\title{
Ultrasonographic diagnosis of unilateral ectopic ureter in a Labrador dog
}

\author{
P A Oglesby ${ }^{\mathrm{a}}$ and A Carter ${ }^{\mathrm{a}}$
}

\begin{abstract}
A 3-month-old, Labrador bitch presented with urinary incontinence. A unilateral, ectopic ureter was diagnosed with ultrasound without the aid of additional contrast radiographic techniques. Ipsilateral hydroureter and mild hydronephrosis were also present. At the level of the bladder trigone the ectopic ureter became intramural and this was clearly demonstrable with ultrasound. The opening of the ectopic ureter into the proximal urethra was, however, not visualised owing to its intrapelvic location.
\end{abstract}

Key words: ectopic ureter, intramural, ultrasound.

Oglesby P A, Carter A Ultrasonographic diagnosis of unilateral ectopic ureter in a Labrador dog. Journal of the South African Veterinary Association (2003) 74(3): 84-86 (En.). Fourways Veterinary Hospital, PO Box 68159, Bryanston, 2021 South Africa.

\section{INTRODUCTION}

An ectopic ureter is a congenital abnormality whereby the ureter opens at a site other than the trigone of the urinary bladder. ${ }^{2}$ This communication describes the diagnosis and findings associated with a unilateral ectopic ureter in a Labrador bitch by means of ultrasound. Emphasis is placed on the ultrasonographic images of the intramural segment of the ectopic ureter at the level of the bladder trigone.

\section{CASE HISTORY}

A 3-month-old Labrador bitch was referred to Fourways Veterinary Hospital in 2002 with a history of urinary incontinence. The owners complained that the bitch had been incontinent since purchasing her as an 8-week-old puppy.

The clinical examination was normal except for mild perivulvar dermatitis. Neither dysuria nor stranguria were evident. Urine was collected by cystocentesis. The specific gravity was measured with a refractometer and found to be 1.020. A dipstick measured the urine $\mathrm{pH}$ at 6 and showed the urine to be negative for protein, blood, ketones and glucose. The sediment contained neutrophils and bacterial rods, indicating cystitis, but this was not confirmed by culture because the patient was later euthanased.

Blood urea nitrogen and creatinine levels were tested with an in-house Vet Test 8008 blood chemistry analyser (IDEXX Laboratories Inc., Maine, USA) and found to be normal.

The patient was sedated using intrave${ }^{a}$ Fourways Veterinary Hospital, PO Box 68159, Bryanston, 2021 South Africa. E-mail: trish@ intekom.co.za alaincarter@fourwaysvet.co.za

Received: April 2003. Accepted: August 2003. nous Medetomidine (Domitor; Novartis) at a dose of $0.03 \mathrm{~m} / \mathrm{kg}$. The abdomen was clipped using an Oster blade and coated with ultrasound gel. The entire abdomen was scanned with the patient in dorsal recumbency using a Sonoline Adara ultrasound machine (Siemens, Halfway House) and an 8.5 MHz linear array transducer. No abnormalities other than in the urinary system were observed.

Both kidneys were scanned in sagittal and transverse planes. They appeared to be of equal and normal size but were unfortunately not measured. The architecture of the right kidney appeared normal but the left kidney showed poor cortico-medullary differentiation due to hypoechogenicity of the renal cortex. The left renal pelvis was mildly dilated and anechoic. The exact dimension of the pelvic dilation was not measured but was suggestive of a mild hydronephrosis.

The right ureter was not visualised. The left ureter, however, was tortuous and dilated to $7 \mathrm{~mm}$ in diameter. Greater than $3 \mathrm{~mm}$ is considered enlarged. ${ }^{10}$ Owing to this dilation it was possible to follow the left ureter in both sagittal and transverse planes from its origin at the renal hilus to the level of the bladder trigone.

Peristalsis was seen in the distal segment of the ureter but neither of the ureteral jets were observed.

The opening of the ureter into the proximal urethra could not be demonstrated ultrasonographically because of the intrapelvic nature of this location. However, in the area of the bladder trigone, the distal ureter was visible as a well-marginated, thin-walled, ovoid structure with anechoic content (Figs 1, 2). This represents the intramural segment of the ectopic ureter, which tunnels into the submucosa of the dorsolateral bladder wall and bypasses the trigone instead of correctly opening into the bladder at this site. The ureteral wall of the intramural segment appeared thicker than the rest of the ureter because the ultrasound image represents both the wall of the ureter and the bladder submucosa.

The bladder contained anechoic fluid diffusely interspersed with hyperechoic specks, which gravitated ventrally when not disturbed. These correspond to the sediment of neutrophils and bacteria found on urine analysis. The bladder wall was not thickened.

Surgery was recommended but the owners opted for euthanasia. On post mortem the diagnosis of unilateral ectopic ureter was confirmed. The intramural segment of the ectopic ureter within the submucosa of the dorsolateral bladder wall is shown in Fig. 3. This corresponds to the ultrasound images of Figs 1 and 2 . In Fig 3, the bladder wall has been exteriorised and deflected to demonstrate the intramural ureteral segment. An iatrogenic longitudinal slit was made into the lumen of the intramural ureter.

\section{DISCUSSION}

Canine ectopic ureter is a congenital abnormality resulting in the opening of the ureter at a site other than the trigone of the urinary bladder. ${ }^{2}$ Alternative sites for ureter termination include the bladder neck, urethra, vagina, uterus and vas deferens. ${ }^{1}$

The overall incidence of ectopic ureter in the canine population is low $(0.016 \%)^{2}$ and bilateral ectopia is present in approximately $25 \%$ of cases. ${ }^{1}$ Females are twenty times more likely to be diagnosed because they invariably present with urinary incontinence. ${ }^{3,12}$ The male dog, however, can appear to urinate normally due to retrograde filling of the bladder if the ureter opens proximal to the external urethral sphincter. ${ }^{5,9,13}$

Ectopic ureters are classified as either extramural or intramural. Extramural ectopic ureters bypass the urinary bladder with no anatomical attachment. Intramural ectopic ureters attach to the dorsal or dorsolateral surface of the urinary bladder, but instead of opening into the trigone, 


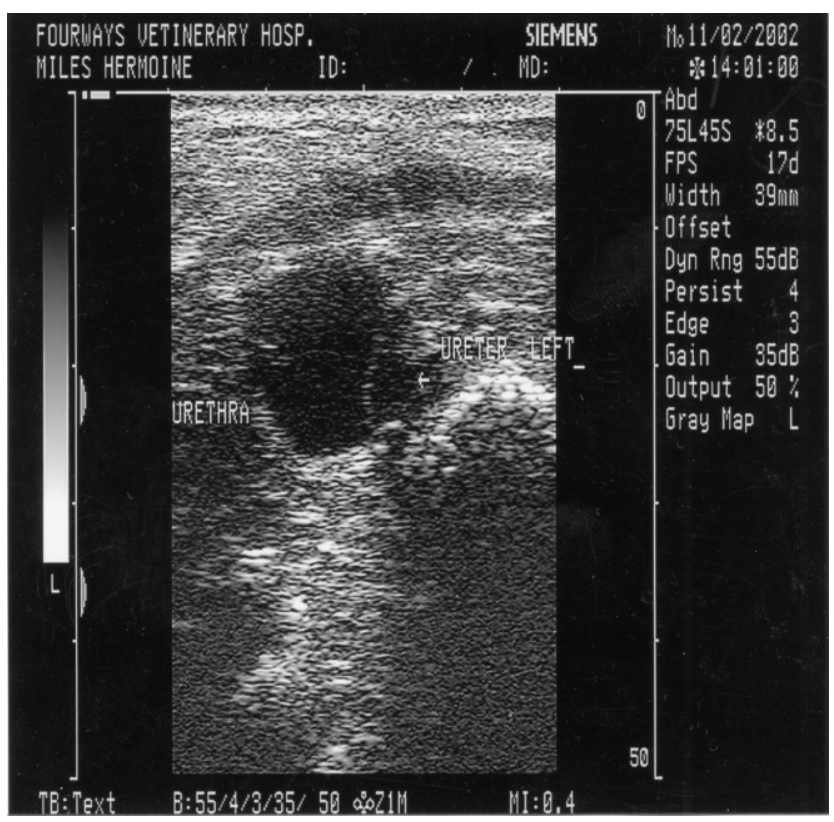

Fig. 1: Transverse ultrasonogram showing a well-marginated, thin-walled, ovoid structure at the bladder trigone. This represents the markedly dilated, intramural segment of the distal ectopic ureter and should not be confused with a ureterocoele. The gas-filled colon lies to the left of the ureter. The label 'urethra' is incorrect and should be ignored.

tunnel distally in the submucosa before opening into the bladder neck, urethra or vagina. $^{11}$

Various breeds have been described as having a predisposition towards ectopic ureters. These include the Siberian husky, Miniature and Toy poodles, Golden retriever, Labrador retriever, Shetland sheepdogs, Spaniels, West highland white terrier, Wire fox terrier, Bulldog and Newfoundland. ${ }^{4,5}$

Urinary incontinence is the major clinical sign associated with ectopic ureter. ${ }^{35}$ Cystitis, perivulvar dermatitis secondary to urine scalding and pyelonephritis from ascending infection may also be present. ${ }^{11}$ Haematology and biochemistry are usually normal.

Other disease entities that may cause urinary incontinence include cystitis, cystic or urethral calculi, a pelvic bladder, persistent urachal diverticulum, ureterocoele, urethral sphincter incompetence, neoplasia, prostatic disease and neurogenic incontinence. ${ }^{12}$

Excretory urography (EU) has previously been the gold standard of diagnosing ectopic ureter. However, there are several disadvantages to this procedure. The patient must be fasted and given an enema prior to the procedure. General anaesthesia and multiple radiographs are required which render EU both timeconsuming and expensive. More significantly, it does not always allow the exact termination of the ureter to be determined. Holt et al. ${ }^{5}$ reported that the precise location of the ureteral opening was

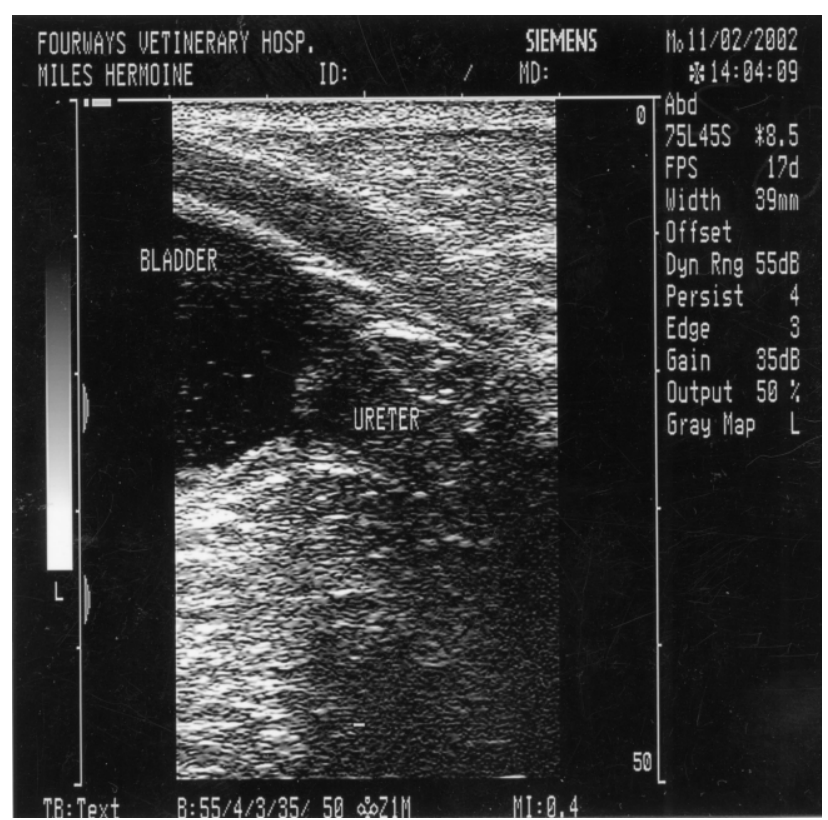

Fig. 2: Sagittal ultrasonogram of the bladder in the region of the bladder trigone. The dilated, intramural ectopic ureter can be seen as a thin-walled, well-marginated, ovoid structure with anechoic content lying at the trigone. There are numerous, pinpoint, hyperechoic specks diffusely interspersed throughout the bladder. These are cells in suspension from a urinary sediment, which indicates that a secondary cystitis is present. demonstrated by EU in only 17 out of 26 cases. This may be caused by poor renal excretion of contrast medium or lack of opacity of the distal ureter due to peristalsis. ${ }^{7}$ The opening of the ectopic ureter may also be obscured by superimposition of the colon, pelvis or contrast medium in the bladder. ${ }^{12} \mathrm{EU}$, therefore, often has to be combined with cystoscopy or additional retrograde contrast radiographic techniques such as double-contrast cystography, urethrovaginography, retrograde urethrography or a micturating

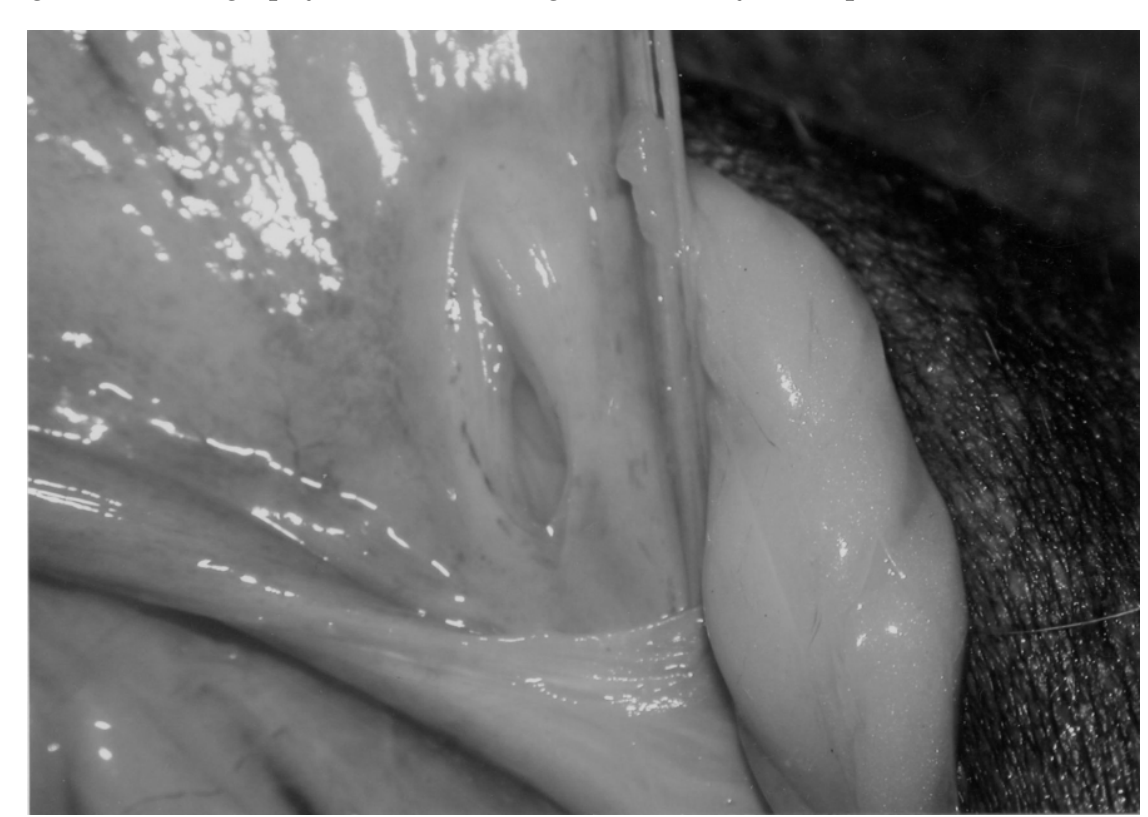

Fig. 3: Post mortem photograph of the intramural segment of the ectopic ureter at the level of the bladder trigone. The bladder wall has been exteriorised and deflected. There is an iatrogenic longitudinal slit into the lumen of the intramural, ectopic ureter. This intramural segment corresponds to the ultrasound images in Figs 1 and 2. 
chronic pyelonephritis or by obstruction of the ureter from a stenotic or absent ureterovesicular junction or intramural ureter segment. $^{10}$

Ureteral jets, which represent the turbulent flow of urine into the bladder, may be observed ultrasonographically as a series of small ecogenic foci streaming periodically from the ureterovesicular junction. ${ }^{7}$ These can be visualised in the normal animal using a high frequency transducer. Colour-flow Doppler is helpful but not essential. ${ }^{6}$ However, if the bladder is displaced caudally, ureteral jets may not be visible owing to their intrapelvic location. ${ }^{7}$ The visibility of ureteral jets can be enhanced if the specific gravity of ureteral and bladder urine differs. This can be achieved by catheterising and filling the bladder with saline or administering a diuretic. ${ }^{7}$ The absence of one or both ureteral jets can therefore be used as an adjunct in the diagnosis of uni-or bilateral ectopic ureters. Neither technique was implemented during the ultrasound examination of this patient, which may explain why the ureteral jet corresponding to the normal ureterovesicular junction was not observed.

In this study the intramural component of the ectopic ureter was clearly visible on ultrasound as a well-marginated, thinwalled, ovoid structure with anechoic content at the bladder trigone. The most important differential diagnosis for this ultrasound image is a ureterocoele which, with ultrasound, will also appear as a thin-walled, round structure containing anechoic fluid. ${ }^{14}$ A ureterocoele is a congenital abnormality that is a focal dilatation of the distal ureter, usually that portion of the ureter which lies within the bladder wall (intravesical) or they may be associated with an ectopic ureter (ectopic) ${ }^{8}$
Post mortem confirmed that the patient did not have a ureterocoele and that the ultrasound images depicted in Figs 1 and 2 , although similar to those that can be expected with a ureterocoele, demonstrated the intramural component of the dilated ectopic ureter.

Ultrasound is a practical diagnostic test for ectopic ureter in small animals. The presence of a dilated ureter passing caudal to its normal opening at the bladder trigone is diagnostic. The absence of a ureteral jet further aids the diagnosis. Furthermore, the urinary system can systematically be scrutinised for concomitant abnormalities associated with ectopic ureter. The kidneys can be examined for pyelonephritis and hydronephrosis. The bladder can be evaluated for size, location, content and bladder wall thickness. The dilated ureter can be examined for ureteral wall thickening, terminal branching, intramural segments, ureterocoele and diverticula. Ultrasound has a comparable accuracy to contrast radiography in diagnosing ectopic ureter but contrast radiography is not as helpful in identifying ureteral morphology. An ectopic ureter may appear on radiography to bypass the bladder where in fact it follows an intramural path. ${ }^{10}$ This study showed that an intramural segment of an ectopic ureter can be identified by ultrasound. Caremust be taken, however, not to confuse this intramural segment with a ureterocoele.

\section{REFERENCES}

1. Carleton S 1992 Canine ectopic ureter: a case example. Veterinary Technician 13: 624-627

2. Dean PW, Constantinescu G M 1988 Canine ectopic ureter. Compendium on Continuing Education for the Practicing Veterinarian 10: 146-157

3. Hayes H M 1974 Ectopic ureter in dogs: epidemiologic features. Teratology 10: 129-132

4. Hayes H M 1984 Breed associations of canine ectopic ureter: a study of 217 female cases. Journal of Small Animal Practice 25: 501-504

5. Holt P E, Gibbs C, Pearson H 1982 Canine ectopic ureter - a review of twenty-nine cases. Journal of Small Animal Practice 23: 195-208

6. Lamb C R, Gregory S P 1994 Ultrasonography of the ureterovesicular junction in the dog: a preliminary report. Veterinary Record 134: 36-38

7. Lamb C R, Gregory S P 1998 Ultrasonographic findings in 14 dogs with ectopic ureter. Veterinary Radiology and Ultrasound 39: 218-223

8. Lautzenhiser S J, Bjorling D E 2002 Urinary incontinence in a dog with an ectopic ureterocele. Journal of the American Animal Hospital Association 38: 29-32

9. Lennox J S 1978 A case report of unilateral ectopic ureter in a male Siberian husky. Journal of the American Animal Hospital Association 14: 331-336

10. Mason L K, Stone E A, Biery D N, Robertson I, Thrall DE 1990 Surgery of ectopic ureters: pre-and postoperative radiographic morphology. Journal of the American Animal Hospital Association 26: 73-79

11. McLoughlin M A, Chew D J 2000 Diagnosis and surgical management of ectopic ureters. Clinical Techniques in Small Animal Practice 15: 17-24

12. Silverman S, Long C D 2000 Diagnosis of urinary incontinence and abnormal urination in dogs and cats. Veterinary Clinics of North America: Small Animal Practice 30: 427-447

13. Smith C W, Stowater J L, Kneller S K 1980 Bilateral ectopic ureter in a male dog with urinary incontinence. Journal of the American Veterinary Medical Association 177: 1022-1024

14. Stiffler K S, McCrackin Stevenson M A, Mahaffey M B, Howerth E W, Barsanti J A 2002 Intravesical ureterocele with concurrent renal dysfunction in a dog: a case report and proposed classification system. Journal of the American Animal Hospital Association 38: 33-39 